\title{
Taxonomic resolution in parasite community studies: are things getting worse?
}

\author{
R. POULIN* and T. L. F. LEUNG \\ Department of Zoology, University of Otago, P.O. Box 56, Dunedin 9054, New Zealand \\ (Received 6 April 2010; revised 13 May 2010; accepted 13 May 2010; first published online 12 fuly 2010)
}

\begin{abstract}
SUMMAR Y
Species identification is crucial for studies of parasite diversity, yet most surveys include taxa identified only to genus or family level. Using a large dataset comprising 950 surveys of helminth communities from 650 different species of fish, bird and mammal hosts, we investigated what determines the level of taxonomic resolution achieved. Identification of all helminths down to species level was achieved in only one-third of surveys, whereas all taxa were identified at least to genus level in two-thirds of surveys. The species richness of a parasite community and past study effort on the host species did not correlate with taxonomic resolution. However, the higher the proportion of parasite taxa occurring as larvae in a community, the lower the resolution achieved. Also, taxonomic resolution was better overall for communities in birds or mammals than for those in fish, and better for trematodes and acanthocephalans than for cestodes and nematodes. Perhaps the most intriguing result was a clear significant effect of year of publication on the taxonomic resolution achieved in parasite surveys: the proportion of helminth taxa identified at least to genus level has remained high until the year 2000, but has dropped in studies published since then. The loss of expertize in parasite taxonomy happening worldwide is one possible explanation. This downward trend needs to be reversed if we are to monitor new parasite occurrences in the face of environmental changes.
\end{abstract}

Key words: species identification, diversity, helminths, vertebrate hosts, larval stages, study effort.

\section{INTRODUCTION}

The worldwide loss of expertise in parasite taxonomy and classical (i.e. morphological, as opposed to molecular) systematics has been identified as one of the greatest challenges for research in parasite biodiversity, ecology and epidemiology (Brooks and Hoberg, 2000, 2001; Poulin and Morand, 2000, 2004). As the current group of experts gradually retire from their university, museum or government positions, they are not being replaced at a matching rate, and so the international pool of parasite taxonomists is rapidly shrinking. The advent of DNA barcoding cannot compensate for this loss of expertise; DNA barcodes can distinguish between 2 or more species, but they tell us nothing about the morphology, ecology or evolutionary history of the species whose genes are sequenced if these have not previously been the subject of a detailed descriptive study (Moritz and Cicero, 2004). Our ability to put a name on any species encountered and link it with a suite of unique phenotypic properties will set the limits of our understanding of the biodiversity and functioning of natural systems.

Most biodiversity surveys of parasites are carried out at the component community level, i.e. all the parasite species exploiting a host population. In

* Corresponding author. Tel: +643479 7983. Fax: +6434797584. E-mail: robert.poulin@stonebow.otago. ac.nz studies of parasite communities of vertebrates, parasite taxa comprising the list found in a given sample of hosts are rarely all identified to the species level. Typically, some are identified only to the genus level, or sometimes only to the family (or even higher) level. Assuming that the authors of these many studies have distinguished correctly among the different species based on their morphology, regardless of whether or not they could assign names to all of them, their estimate of the species richness of the community should nevertheless be correct. In recent years, however, the use of molecular tools has revealed that previously named and described species of parasites often consist of 2 or more cryptic species (e.g. Hung et al. 1999; Jousson et al. 2000; Blouin, 2002; Poulin and Keeney, 2008; Leung et al. 2009; Blasco-Costa et al. 2010). Therefore some of the parasite species named in community lists are likely to include cryptic species; this possibility should be even greater for taxa identified only down to genus or family level, since the distinguishing features of these unnamed taxa are unknown. Poor taxonomic resolution may thus mask undetected biodiversity, and could lead to biases in ecological analyses where accurate estimates of species richness or species cooccurrences matter.

A quick glance at published studies suggests that taxonomic resolution is not the same across surveys of parasite component communities. There are many possible reasons for this. Resolution could be greater 
in some higher taxa of hosts or parasites, possibly because of the activities of a few prolific taxonomists working on those taxa. We would expect a relatively constant proportion of unnamed species in all species lists, regardless of their length, though there may be more unnamed species in rich communities because of a larger component of rare (and undescribed) species. Because most parasite species are known only from their adult stages, communities consisting of many larval parasites may therefore include a greater proportion of unnamed species. We might also expect the parasite communities of well-studied host species, such as domestic animals and those that are broadly distributed or of commercial importance, to be better known than those of host species that have been the subject of less scientific research. Finally, given that both our knowledge of parasite taxonomy and the number of described species increase over time, we may also expect that more recent studies provide better-resolved lists of parasite species than earlier ones. Although these and other factors are all likely to correlate with the taxonomic resolution of parasite surveys, the possibility that they systematically affect taxonomic resolution remains untested.

Here, we search for correlates of taxonomic resolution across studies of parasite communities of vertebrates. We focus exclusively on helminth endoparasites (trematodes, cestodes, nematodes and acanthocephalans) of fish, birds and mammals, since these have been the subject of numerous investigations. We address the following questions. (i) Is taxonomic resolution better for certain types of hosts or for certain higher taxa of parasites than others? (ii) Is taxonomic resolution relatively lower for species-rich communities than for those with fewer species? (iii) Is taxonomic resolution worse when the community includes many species occurring at their larval stage, such as the metacercariae of trematodes or the plerocercoids of cestodes? (iv) Is taxonomic resolution dependent on how much past research has been conducted on parasitism in the host species? (v) Is taxonomic resolution improving or worsening over time? Our analyses are exploratory in nature rather than aimed at testing specific hypotheses, and our goal is to identify factors associated with the level of taxonomic resolution that may bias the results of ecological research on parasites.

\section{MATERIALS AND METHODS}

We gathered data from published surveys of helminth communities in vertebrates, obtained by combining the studies already compiled by Poulin (1995), and all other relevant studies available through the University of Otago library and found in a search of the ISI Web of Knowledge using the search string: '(parasit* or helminth*) and (fish* or bird* or mammal*) and (fauna or communit*)'. Although this does not guarantee that all pertinent studies were included in our analyses, this compilation should yield a large, representative and unbiased sample of studies published from several decades ago to the present. We only included studies that provided a clear list, normally in table format, of all species of trematodes, cestodes, nematodes and acanthocephalans found in 1 sample of hosts from the same species. When data were provided in the same study on 2 or more samples of the same host species collected at different times or from different localities within a defined region, we only used the total species list for all samples combined.

Each entry in our dataset corresponds to 1 parasite survey for 1 host species from 1 study, i.e. 1 local parasite community; some host species have been the subject of a few separate studies, and thus for those host species there are 2 or more entries in the dataset. For each entry, we recorded: (i) the name of the host species; (ii) the host type, i.e. fish, bird or mammal, and the order to which the host species belonged; (iii) the total number of trematode, cestode, nematode and acanthocephalan species, whatever their level of taxonomic resolution; (iv) for each group of parasites as well as for all taxa in a survey, the proportion of 'species' identified to the species level (binomial Latin name), and the proportion identified at least to the genus level; (v) the proportion of parasite species occurring not as adults but as larvae or juveniles; (vi) the year in which the survey was published; and (vii) the relative study effort devoted to parasites in the host species, estimated as the total number of records obtained from a search of the ISI Web of Science using the keywords: Latin binomial name of host species AND (parasit* OR helminth* OR tremat* OR digenea* OR nemat* OR cestod* or acanthoceph*). Although this last measure may not perfectly capture all previous taxonomic work on the parasites found in the host species, it should nevertheless correlate with the extent to which researchers have studied the parasites of that host. This is also a much more relevant measure of study effort than host sample size, since existing taxonomic knowledge of the parasites in a given host species depends on the extent of previous taxonomic work rather than on how many individual hosts are examined in a particular survey.

Because the proportion of parasite taxa that can be identified down to the genus or species level depends on human knowledge, and is thus not a biological property of a host species, there was no reason to control for host phylogenetic affinities in statistical analyses. Nevertheless, we included host taxonomic order as a variable in the analyses to account for possible higher-order phylogenetic influences. Furthermore, some host species contribute 2 or more entries in the dataset, and to avoid potential pseudoreplication, the analysis was conducted both at the survey level (across all entries in the dataset) and at the host species level. For the latter analysis, for 
Table 1. Summary of the data on 950 surveys of helminth communities in vertebrate hosts, and on the past study effort devoted to parasitism in those hosts

\begin{tabular}{lllll}
\hline \hline Host type & $\begin{array}{l}\text { Number of } \\
\text { helminth } \\
\text { surveys }\end{array}$ & $\begin{array}{l}\text { Median helminth species } \\
\text { richness (interquartile range) }\end{array}$ & $\begin{array}{l}\text { Number of } \\
\text { host species }\end{array}$ & $\begin{array}{l}\text { Median study effort* } \\
\text { (interquartile range) }\end{array}$ \\
\hline Fish & 419 & $8(4-12)$ & 323 & $4(1-15)$ \\
Birds & 161 & $10(5-16)$ & 118 & $6(3-15)$ \\
Mammals & 370 & $8(5-13)$ & 209 & $8(3-21)$ \\
\hline \hline
\end{tabular}

* Number of previous studies on parasites per host species, from a search of the Web of Science.

each host species with 2 or more entries in the dataset, only the survey with the highest number of parasite species was retained as the most representative of the parasite fauna of that host species; other entries for that host were excluded from the 'species-level' analyses.

There were therefore 2 levels of analysis (across all surveys, and across host species) for each of 2 measures of taxonomic resolution (proportion of taxa identified to species level, and proportion identified at least to genus level), giving a total of 4 versions of the analysis of the determinants of taxonomic resolution in surveys of parasite communities. For each of these 4 analyses, we used generalized linear models (GLM) implemented with the statistical software JMP 7.0. Since the response variable was always a proportion, i.e. the proportion of species identified either to species or at least to genus level, all models had a binomial error structure and logit link function. The explanatory variables included in the starting models were: host type (fish, bird or mammal), host order (nested within host type), parasite species richness (or the total number of helminth taxa in the community), the proportion of taxa occurring as larvae, study effort, and year of publication. Certain variables required either logarithmic (parasite species richness, study effort) or arcsine (proportion of taxa occurring as larvae) transformation prior to analysis. After starting with a full model, significance levels were based on the deviance explained by each factor, based on $\chi^{2}$ statistics, following backward stepwise elimination of non-significant $(P>0 \cdot 05)$ terms. Likely two-way interactions were initially included, but none were significant. Only the final models are shown in the results.

In addition, to determine whether taxonomic resolution is generally better for certain higher taxa of parasites than others, a couple of two-way ANOVAs were performed across all surveys, one with the proportion of taxa identified to species level as response variable, and the other with the proportion of taxa identified to at least genus level. Although they are proportions, these variables did not require transformations based on inspection of the residuals. The 2 factors considered were host type (fish, bird or mammal) and parasite group

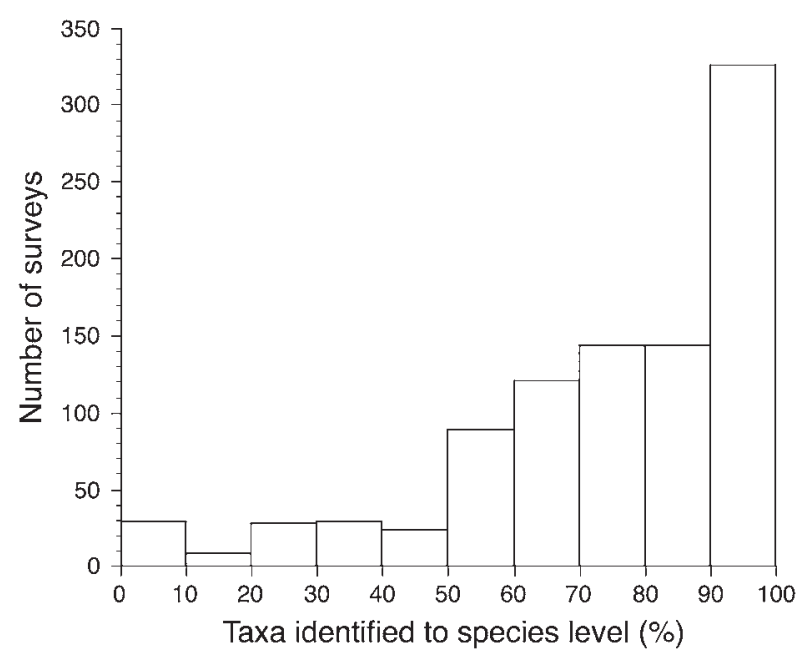

Fig. 1. Frequency distribution of taxonomic resolution values among 950 surveys of helminth communities in fish, bird and mammal hosts. Taxonomic resolution is measured as the percentage of parasite taxa in a community that were identified to species level.

(trematodes, cestodes, nematodes, or acanthocephalans). For this analysis, each survey contributed more than 1 data point, since each of the 4 parasite groups, if present, were treated separately.

\section{RESULTS}

Data were obtained for a total of 950 helminth communities from 650 different host species surveyed between the years 1936 and 2009; the full data set, including the list of the original 545 published sources, is available as Supplementary Material (in online version only). There were fewer surveys of helminth communities, and these involved fewer host species, in bird hosts than in mammals and fish (Table 1). Helminth communities tended to be slightly more species-rich in birds than in other host types, and past study effort on parasitism in given host species tended to be higher for mammals, but these differences were quantitatively small ('Table 1). Overall, identification of $100 \%$ of helminth taxa down to species level was achieved in only about one-third of the surveys, with the median value being $78 \cdot 8 \%$ (Fig. 1). In contrast, taxonomic resolution to 
Table 2. Results of generalized linear models of the factors influencing taxonomic resolution in helminth communities of vertebrates

(Four analyses are shown, at 2 levels (across all surveys, across host species) and for 2 measures of resolution (proportion of taxa identified to species level, proportion identified at least to genus level). The $\chi^{2}$ tests assess the significance of the deviance explained by each factor.)

\begin{tabular}{|c|c|c|c|c|c|c|}
\hline \multirow[b]{2}{*}{ Taxonomic resolution } & \multirow[b]{2}{*}{ Factor } & \multirow[b]{2}{*}{ D.F. } & \multicolumn{2}{|c|}{ Across surveys } & \multicolumn{2}{|c|}{$\begin{array}{l}\text { Across host } \\
\text { species }\end{array}$} \\
\hline & & & $\chi^{2}$ & $P$ & $\chi^{2}$ & $P$ \\
\hline \multirow[t]{2}{*}{ To the species level } & Host type & 2 & $11 \cdot 58$ & $0 \cdot 0031$ & $6 \cdot 12$ & $0 \cdot 0450$ \\
\hline & Proportion of larval taxa & 1 & $14 \cdot 61$ & $0 \cdot 0001$ & $13 \cdot 59$ & $0 \cdot 0002$ \\
\hline \multirow[t]{2}{*}{ To at least genus level } & Proportion of larval taxa & 1 & $19 \cdot 65$ & $0 \cdot 0001$ & $13 \cdot 75$ & $0 \cdot 0002$ \\
\hline & Year of publication & 1 & $4 \cdot 68$ & $0 \cdot 0305$ & $7 \cdot 49$ & $0 \cdot 0062$ \\
\hline
\end{tabular}

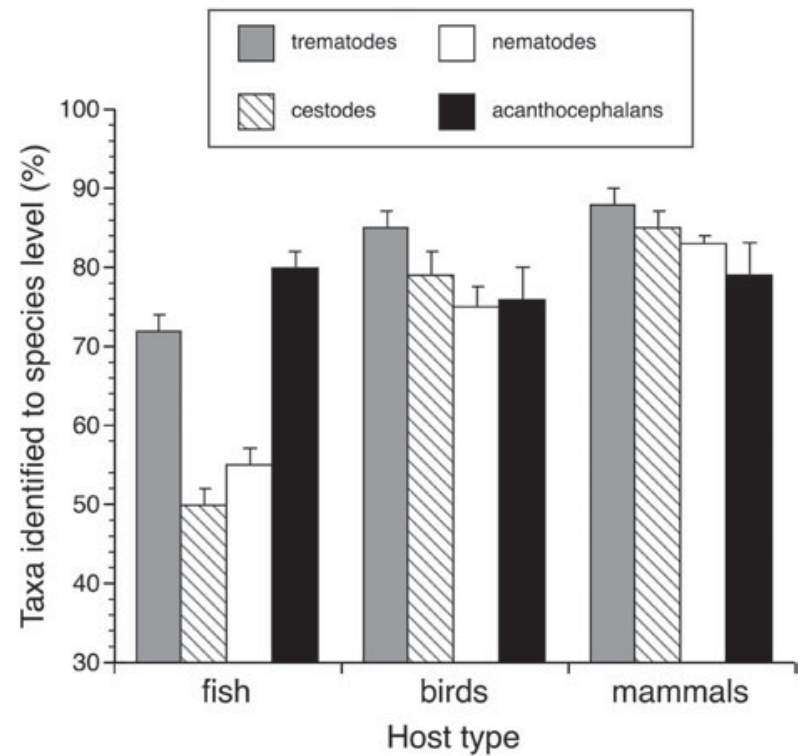

Fig. 2. Mean ( \pm s.E.) taxonomic resolution for 4 groups of helminth parasites in 3 types of vertebrate hosts, fish (419 surveys), birds (161 surveys) and mammals (370 surveys). Taxonomic resolution is measured as the percentage of parasite taxa in the community surveyed that were identified to species level.

the genus level was greater, with identification of $100 \%$ of helminth taxa at least to genus level achieved in about two-thirds of the surveys.

In the GLMs testing for the determinants of taxonomic resolution at the species level, parasite species richness, study effort, host taxonomic order (nested within host type) and year of publication were not retained in the final models. This was true whether the analysis was performed across surveys or across host species (i.e. including a single survey per host species). However, both host type and the proportion of taxa occurring as larval stages significantly correlated with the proportion of species identified to the species level (Table 2). Taxonomic resolution was generally higher for helminth communities surveyed in mammal hosts, followed closely by those in birds, and with communities in fish hosts achieving much lower resolution to the species level

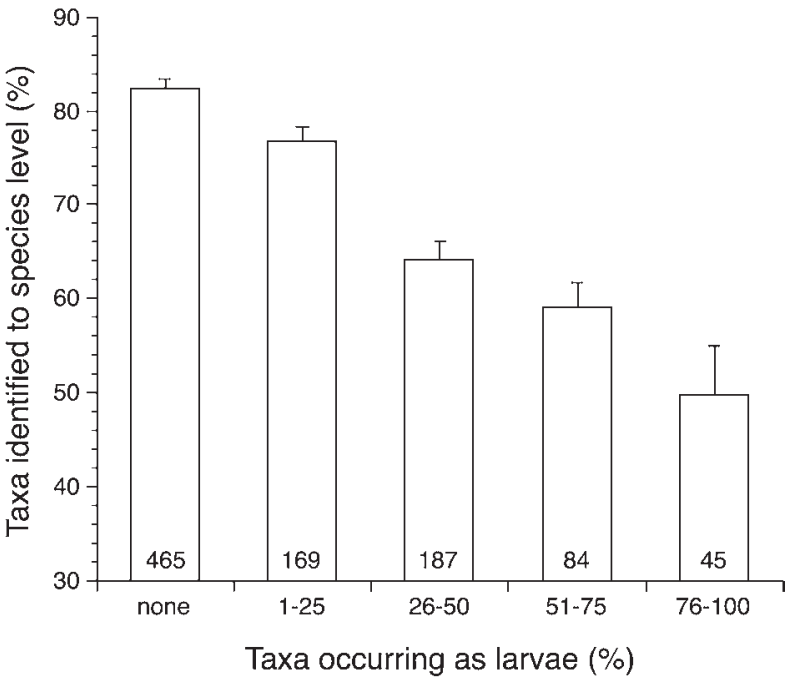

Fig. 3. Mean ( \pm s.E.) taxonomic resolution of parasite taxa in helminth communities of vertebrate hosts, as a function of the percentage of taxa in a community that occurred as larval or juvenile stages. All host types (fish, birds and mammals) are pooled here. Numbers on the bars indicate the number of communities surveyed in each category. Taxonomic resolution is measured as the percentage of parasite taxa in the community surveyed that were identified to species level.

(Fig. 2). The more helminths occurred as larval or juvenile stages in a community, the lower its level of taxonomic resolution (Fig. 3). For instance, in communities consisting of $50 \%$ or more larval helminth taxa, typically only a little over half of the taxa were identified to the species level.

The GLMs testing for the determinants of taxonomic resolution at least to the genus level yielded slightly different results. As above, parasite species richness, study effort, and host taxonomic order (nested within host type) were not retained in the final models, whereas the proportion of taxa occurring as larval stages again proved a significant correlate of taxonomic resolution to the genus level (Table 2). However, whether across surveys or host species, host type was excluded from the final models, whereas year of publication was retained (Table 2). 


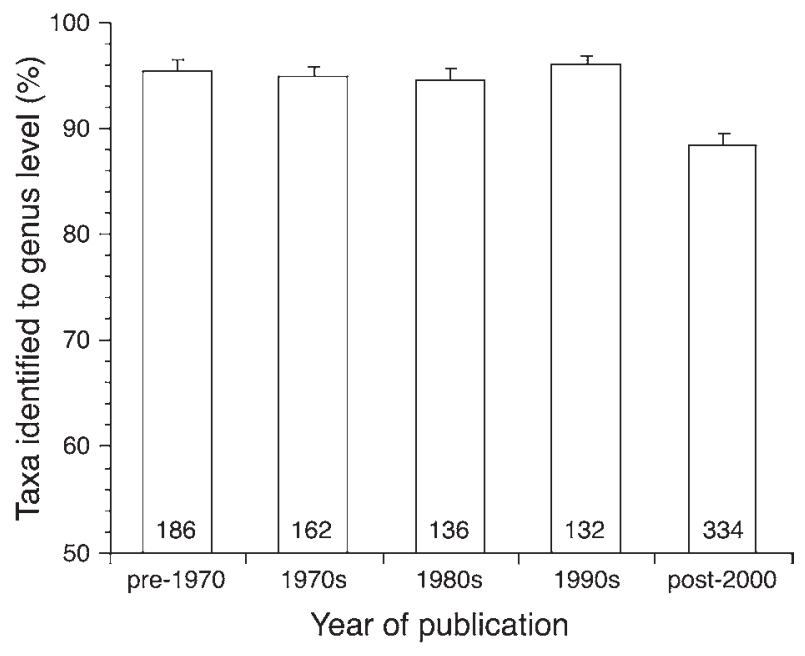

Fig. 4. Mean ( \pm s.E.) taxonomic resolution of parasite taxa in helminth communities of vertebrate hosts, as a function of the year in which the survey was published. Numbers on the bars indicate the number of communities surveyed in each time period. Taxonomic resolution is measured as the percentage of parasite taxa in the community surveyed that were identified at least to genus level.

The data indicate that the proportion of helminth taxa identified at least to genus level has remained high, at around $95 \%$ on average, until the year 2000 , but has dropped to an average of $88 \%$ in studies published since that time (Fig. 4). This effect of year of publication is independent of host type; nevertheless, it is worth nothing that the proportion of surveys on fish hosts has been higher since the year $2000(64 \%)$ than it was prior to that time (33\%).

Finally, the two-way ANOVA indicated that the proportion of taxa identified to species level across surveys was influenced by parasite group $\left(F_{3,2725}=\right.$ $15 \cdot 20, P<0 \cdot 0001)$, host type $\left(F_{2,2725}=82 \cdot 97, P<\right.$ $0 \cdot 0001)$, and the interaction between these factors $\left(F_{6,2725}=11 \cdot 95, P<0 \cdot 0001\right)$. Whatever the host type, taxonomic resolution was greater for trematodes than for other helminths (Fig. 2). Also, whatever the host type, on average $75-80 \%$ of acanthocephalans in the surveys were identified to the species level; the other 3 helminth groups generally achieved that or a higher level of resolution in bird and mammal hosts (Fig. 2). However, this was not the case in fish hosts, particularly for cestodes and nematodes of which only about half were assigned a species name (Fig. 2). Essentially the same results were obtained when the ANOVA was repeated using a different level of taxonomic resolution, i.e. to at least genus level instead of to species level, as response variable (both factors and their interaction, $P<0.0001)$.

\section{DISCUSSION}

The loss of expertise in parasite taxonomy has been highlighted previously (Brooks and Hoberg, 2000,
2001). It is an unfortunate consequence of changing funding priorities in science worldwide (e.g. Cribb, 2004). In addition, taxonomic studies are time consuming and lead to publications in journals with relatively low impact factors, making them unattractive for younger researchers seeking permanent appointments. Nevertheless, the number of described species keeps rising, and there has been a steady increase in available resources allowing species identification. Our results suggest that the ongoing loss of taxonomic expertise may already have a measurable impact on ecological studies of parasite diversity. We provide evidence that, over the past decade, there has been a significant drop in the proportion of parasite taxa identified at least to genus level in surveys of parasites in vertebrate hosts, relative to surveys published earlier. Of course, this decrease in taxonomic resolution is not necessarily due to a smaller worldwide pool of taxonomic experts. It could also reflect lower standards of quality, i.e. a decrease in the effort invested by researchers into species identification, and an acceptance of this lower effort by scientific journals. An alternative explanation could be that in recent years, surveys have targeted host species from new geographical areas about which less is known in terms of parasite taxonomy. This last explanation seems unlikely, however, since there is no significant correlation between year of publication and logtransformed study effort among surveys in our dataset $(r=-0 \cdot 033, N=950, P=0 \cdot 308)$. Our analyses reveal a worrying trend, though further research will be necessary to identify its cause.

Whatever its underlying cause, the clear effect of year of publication seen in our generalized linear model, and the large sample sizes on which this finding is based, are cause for concern for the present and future of ecological parasitology. It has become increasingly apparent that poor taxonomic resolution often undermines pattern analysis in other areas of ecology and biodiversity research. For instance, in food web research, the level of taxonomic resolution achieved has a significant impact on the measurement of basic food web properties (Martinez, 1991; Thompson and Townsend, 2000). Taxonomic resolution is also important in biogeographical studies. For example, identification of pollen down to species level can reveal shifts in plant assemblages that are not apparent with lower resolution (Finkelstein et al. 2006). In studies of environmental impact assessment, including many investigations of the impacts of pollution on aquatic habitats, low levels of taxonomic resolution can obscure important changes to the biota (Terlizzi et al. 2003, 2009; Jones, 2008). Although coarse taxonomic resolution may suffice in certain cases, poor resolution generally fails to account for total biodiversity and can limit the usefulness of any dataset (Anderson et al. 2005). Conclusions of ecological studies on parasite diversity 
or distribution may be equally subject to errors arising from poor taxonomic resolution if species identification frequently stops at the genus or family level. For instance, several recent studies have examined the influence of geographical distance on the similarity in species composition between parasite communities (Poulin, 2003; Brouat and Duplantier, 2007; Seifertova et al. 2008; Pérez-del-Olmo et al. 2009). In such studies, poor taxonomic resolution can result in inflated estimates of similarity between pairs of communities.

Our analyses have also revealed other factors acting on the level of taxonomic resolution achieved in parasite surveys. Whereas the species richness of parasite communities did not influence the taxonomic resolution achieved in any given study, the taxonomic affiliations of both hosts and parasites influenced the proportion of parasites identified down to species level. Greater taxonomic resolution was achieved for parasite assemblages in birds and mammals than those in fish; this was also apparent when each helminth group was considered separately, except for acanthocephalans where taxonomic resolution was the same regardless of whether the host was a fish, bird or mammal. These patterns may, in part, be due to the fact that more helminths occur in fish as larval stages than in birds or mammals. With rare exceptions (e.g. trypanorhynch cestodes), identification to species, or even genus, level requires the presence of discriminating anatomical features only present in adult worms. In fact, formally naming a new species requires description of adult stages. Fish often serve as intermediate or paratenic hosts for a range of widespread helminth taxa (Anisakis nematodes, metacestodes of cestodes, metacercariae of strigeid trematodes, etc) whose identification to species level based on morphology is generally not possible. Indeed, the proportion of taxa occurring as larval stages was significantly correlated with the proportion of species identified to the species level in our analyses. However, host taxon and the proportion of larval helminths had independent effects, therefore birds and mammals have better-resolved parasite communities regardless of the proportion of larval helminths they harbour. This may be due to the fact that birds and mammals have benefited from greater study effort in terms of parasite investigations, even though the effect of study effort per se was not significant in the GLMs.

The dataset we compiled indicates that the majority, i.e. about two-thirds, of parasite surveys fail to identify all parasites to species level; typically, only about three-quarters of parasites in a survey are given a species name. The drop in taxonomic resolution seen in surveys published in the last decade translates, in absolute terms, to about 1 extra taxon per survey not identified to at least genus level. This may not seem much, but the cumulative effect of this increasing lack of resolution on estimates of parasite diversity can be substantial (Poulin and Morand, 2004). Low taxonomic resolution also hinders any attempt to assess host specificity or transfers of parasites from one host to another, a critical issue in studies of disease risk following biological invasions (Torchin et al. 2002; Kelly et al. 2009). It is therefore imperative that we identify the cause of the recent drop in taxonomic resolution seen in parasite surveys, and find ways to reverse the downward trend.

\section{ACKNOWLEDGEMENTS}

We are grateful to Isabel Blasco-Costa for useful comments on an earlier draft of this manuscript. Financial support came in part via a grant from the Marsden Fund.

\section{REFERENCES}

Anderson, M. J., Connell, S. D., Gillanders, B. M., Diebel, C. E., Blom, W. M., Saunders, J. E. and

Landers, T. J. (2005). Relationships between taxonomic resolution and spatial scales of multivariate variation.

Fournal of Animal Ecology 74, 636-646.

Blasco-Costa, I., Balbuena, J. A., Raga, J. A., Kostadinova, A. and Olson, P. D. (2010). Molecules and morphology reveal cryptic variation among digeneans infecting sympatric mullets in the Mediterranean. Parasitology 137, 287-302.

Blouin, M. S. (2002). Molecular prospecting for cryptic species of nematodes: mitochondrial DNA versus internal transcribed spacer. International Fournal for Parasitology 32, 527-531.

Brooks, D. R. and Hoberg, E. P. (2000). Triage for the biosphere: the need and rationale for taxonomic inventories and phylogenetic studies of parasites. Comparative Parasitology 67, 1-25.

Brooks, D. R. and Hoberg, E. P. (2001). Parasite systematics in the $21^{\text {st }}$ century: opportunities and obstacles. Trends in Parasitology 17, 273-275.

Brouat, C. and Duplantier, J. M. (2007). Host habitat patchiness and the distance decay of similarity among gastro-intestinal nematode communities in two species of Mastomys (southeastern Senegal). Oecologia 152, 715-720.

Cribb, T. H. (2004). Living on the edge: parasite taxonomy in Australia. International Fournal for Parasitology 34, 117-123.

Finkelstein, S. A., Gajewski, K. and Viau, A. E. (2006). Improved resolution of pollen taxonomy allows better biogeographical interpretation of post-glacial forest development: analyses from the North American Pollen Database. Fournal of Ecology 94, 415-430.

Hung, G. C., Chilton, N. B., Beveridge, I., Zhu, X. Q., Lichtenfels, J. R. and Gasser, R. B. (1999). Molecular evidence for cryptic species within Cylicostephanus minutus (Nematoda: Strongylidae). International Fournal for Parasitology 29, 285-291.

Jones, F. C. (2008). Taxonomic sufficiency: the influence of taxonomic resolution on freshwater bioassessments using benthic macroinvertebrates. Environmental Reviews 16, 45-69. 
Jousson, O., Bartoli, P. and Pawlowski, J. (2000). Cryptic speciation among intestinal parasites (Trematoda: Digenea) infecting sympatric host fishes (Sparidae). Fournal of Evolutionary Biology 13, 778-785.

Kelly, D. W., Paterson, R. A., Townsend, C. R., Poulin, R. and Tompkins, D. M. (2009). Parasite spillback: a neglected concept in invasion ecology? Ecology 90, 2047-2056.

Leung, T. L. F., Keeney, D. B. and Poulin, R. (2009). Cryptic species complexes in manipulative echinostomatid trematodes: when two becomes six. Parasitology 136, 241-252.

Martinez, N. D. (1991). Artifacts or attributes: effects of resolution on the Little Rock Lake food web. Ecological Monographs 61, 367-392.

Moritz, C. and Cicero, C. (2004). DNA barcoding: promise and pitfalls. PLoS Biology 2, 1529-1531.

Pérez-del-Olmo, A., Fernández, M., Raga, J. A., Kostadinova, A. and Morand, S. (2009). Not everything is everywhere: the distance decay of similarity in a marine host-parasite system. Fournal of Biogeography 36, 200-209.

Poulin, R. (1995). Phylogeny, ecology, and the richness of parasite communities in vertebrates. Ecological Monographs 65, 283-302.

Poulin, R. (2003). The decay of similarity with geographical distance in parasite communities of vertebrate hosts. Fournal of Biogeography 30, 1609-1615.
Poulin, R. and Keeney, D. B. (2008). Host specificity under molecular and experimental scrutiny. Trends in Parasitology 24, 24-28.

Poulin, R. and Morand, S. (2000). The diversity of parasites. Quarterly Review of Biology 75, 277-293.

Poulin, R. and Morand, S. (2004). Parasite Biodiversity. Smithsonian Institution Press, Washington, DC, USA.

Seifertova, M., Vyskocilova, M., Morand, S. and Simkova, A. (2008). Metazoan parasites of freshwater cyprinid fish (Leuciscus cephalus): testing biogeographical hypotheses of species diversity. Parasitology 135, 1417-1435.

Terlizzi, A., Anderson, M. J., Bevilacqua, S., Fraschetti, S., Wlodarska-Kowalczuk, M. and Ellingsen, K. E. (2009). Beta diversity and taxonomic sufficiency: do higher-level taxa reflect heterogeneity in species composition? Diversity and Distribution 15, $450-458$

Terlizzi, A., Bevilacqua, S., Fraschetti, S. and Boero, F. (2003). Taxonomic sufficiency and the increasing insufficiency of taxonomic expertise. Marine Pollution Bulletin 46, 556-561.

Thompson, R. M. and Townsend, C. R. (2000). Is resolution the solution? The effect of taxonomic resolution on the calculated properties of three stream food webs. Freshwater Biology 44, 413-422.

Torchin, M. E., Lafferty, K. D. and Kuris, A. M. (2002). Parasites and marine invasions. Parasitology 124, S137-S151. 